\title{
Response suppression in the CER paradigm with extinction as the aversive event
}

ARNOLD KAUFMAN, University Wisconsin, Milwaukee, Wis. 53201

A pair of albino rats, responding on a $V I$ 15-sec reinforcement schedule, were exposed in a yoked procedure to responsecontingent and response-independent periods of extinction preceded by a $15-\mathrm{sec}$ warning signal. Only response-contingent extinction produced a pronounced suppressive effect during the warning signal. When extinction was response-independent, some decrement occurred during the warning signal, but this effect was no greater than when the warning signal preceded a period of stimulus change unaccompanied by extinction. The results, interpreted as passive avoidance of the extinction period, support the contention that a period of extinction constitutes an aversive event for a deprived organism, although the pronounced conditioned emotional response usually produced by aversive electric shock was not evident in the present study.

Investigations which have employed the CER paradigm to study the aversive properties of extinction have produced some confusion with respect to their findings. Thus, in a recent study, Leitenberg, Bertsch, \& Coughlin (1968) reported that their finding of response suppression during a preextinction stimulus "corrects a misleading picture in the literature of a secure finding: namely, that time out invariably produces an effect opposite to that of shock in the CER paradigm." Although it is true that most studies utilizing the CER paradigm have reported response acceleration during a preextinction stimulus (Ferster, 1958; Leitenberg, 1966; Trenholme, Baron, \& Kaufman $^{2}$ ), it is important to note that Herrnstein's (1955) pioneering study found that response suppression or acceleration was determined by the response rate established on the positively reinforced baseline schedule. Thus it would have been more correct for Leitenberg et al to say that their results, if reliable, contradict the finding that acceleration to a preextinction stimulus invariably occurs when low-rate producing schedules are used.

Since the confusion concerning the generality of the response acceleration effect to a preextinction stimulus may result from the fact that Hermstein's study was not published, and that no studies have been reported replicating Herrnstein's finding of response suppression to a preextinc- tion stimulus on a high-rate producing schedule, the present investigation was undertaken to provide such a replication. In addition, the present study was designed to determine whether such suppression was attributable to: (1) noncontingent exposure to a period of extinction; (2) response-contingent exposure to extinction; or (3) exposure to stimulus change rather than a period of extinction.

\section{SUBJECTS AND APPARATUS}

The Ss were two female albino rats of the Holtzman strain, 90 days old at the start of the experiment. Throughout the experiment, Ss were kept at $80 \%$ of the weight of a free-feeding weight control group of the same age. Apparatus consisted of two Lehigh Valley sound-attenuated rat chambers, with response lever, liquid dipper, and cue light located on the metal front wall, and chamber light located alongside the clear Plexiglas left wall. White noise was used to mask sounds from electromechanical programming equipment.

\section{PROCEDURE}

Subjects were first trained to press the response lever in the presence of the cue light to receive $.01 \mathrm{ml}$ of sweetened Pet evaporated milk, and then were placed on a 15-sec variable interval schedule for $2 \mathrm{~h}$ a day for Sessions 1-50. During Sessions $51-65$, a stimulus testing phase was programmed in which Ss were exposed to a yoked procedure. Five trials per session were scheduled at irregular intervals, during which the cue light darkened, the chamber light illuminated, and the first response by S1 after $15 \mathrm{sec}$ of chamber-light illumination produced $15 \mathrm{~min}$ of complete darkness for both Ss. Responses by S2 had no effect on the light and dark stimuli. The VI $15 \cdot \mathrm{sec}$ reinforcement schedule remained in effect for both $\mathrm{Ss}$ throughout the session in the presence of all stimuli. For Sessions 66-105 the procedure remained the same except that no reinforcement was available to either $S$ in the 15-min dark periods of each trial. Finally, during Sessions $106 \cdot 145$, the response-contingent basis on which the $15-\mathrm{min}$ extinction periods were programmed was reversed for the Ss. Again, the procedure remained as before except that extinction was now programmed for both Ss following the first response by $\mathrm{S} 2$ after $15 \mathrm{sec}$ of chamberlight illumination. In these sessions, responses by $\mathrm{Sl}$ had no effect on the programming of extinction periods.

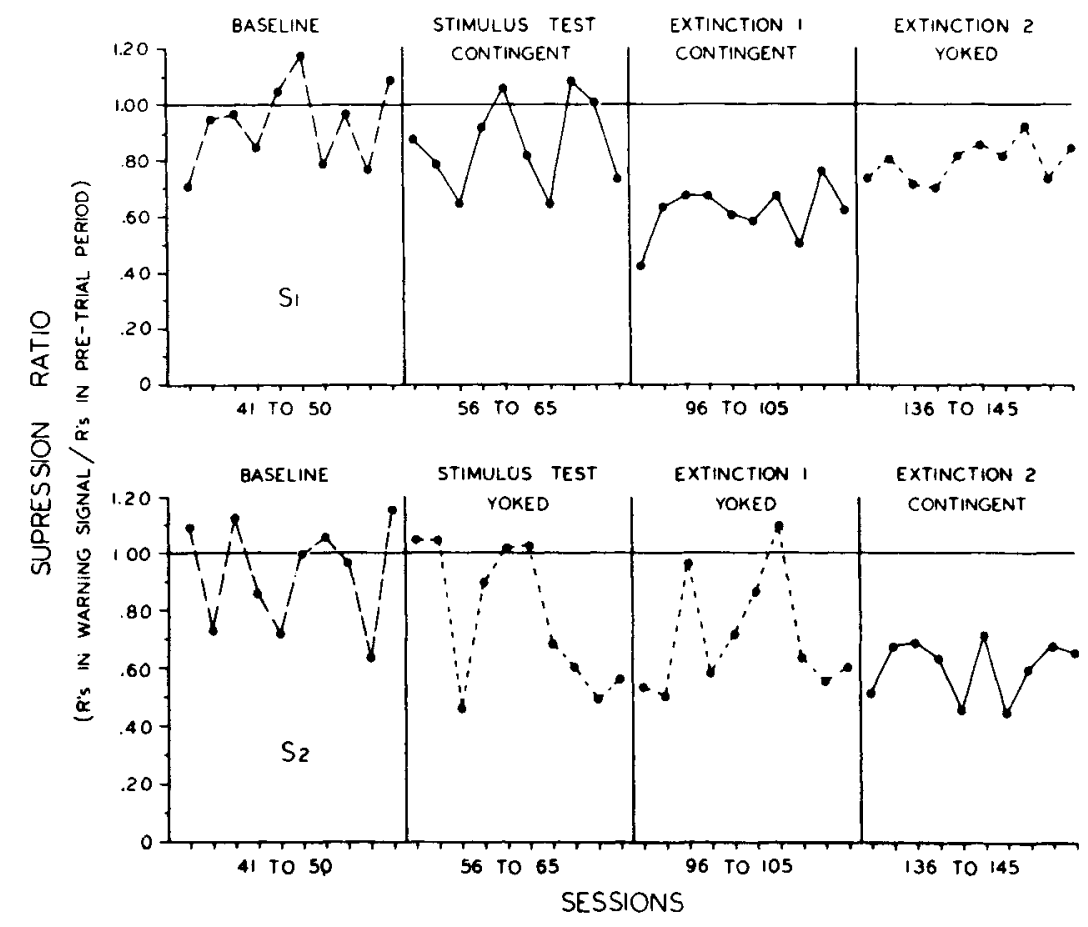

Fig. 1. Suppression ratios for the CER procedure with extinction as the aversive event. 
Table 1

Number and Percentage of Last 10 Days* in which Suppression Ratio Fell Above and Below .70

\begin{tabular}{lcccc}
\hline & Baseline & $\begin{array}{c}\text { Stimulus } \\
\text { Test }\end{array}$ & $\begin{array}{c}\text { Extinction } \\
\text { Yoked }\end{array}$ & $\begin{array}{c}\text { Extinction } \\
\text { Contingent }\end{array}$ \\
\hline Above .70 & $19-95 \%$ & $13-65 \%$ & $14-70 \%$ & $2-10 \%$ \\
Below .70 & $1-5 \%$ & $7-35 \%$ & $6-30 \%$ & $18-90 \%$ \\
\hline
\end{tabular}

* Data for both animals is combined, totaling 20 measures for cach 10-day period.

\section{RESLLTS}

In all sessions, recordings of response frequency were made for the total session, the $15 \mathrm{sec}$ preceding each trial (pretrial period), the first $15 \mathrm{sec}$ of chamber-light illumination (the warning signal), and the 15-min periods when either darkness alone or darkness with extinction was progranmed. For Sessions 1-50 (baseline), these recordings were made during the portions of the session in which the trials would later be programmed. but no trials were presented.

Response frequencies were converted to daily suppression ratios obtained by dividing the total number of responses during the warning signal by the total number of responses during the pretrial periods. Figure 1 presents the daily suppression ratios for each $\mathrm{S}$ for the last 10 days of each procedure. It is apparent that no systematic trend in responding was present during the baseline sessions $(41-50)$. while introduction of the stimuli without extinction conditions present during the dark period produced a slight tendency toward suppression in the presence of the warning signal for both animals (Sessions 56-65). When extinction was introduced during the dark stimulus (Sessions 9(-105), it is evident that S1, the animal upon whose response the extinction period was contingent, showed a much more consistent pattern of suppression during the preextinction warning signal, while the yoked control continued the pattern of erratic suppression exhibited in the stimulus testing sessions. Finally, the effect of reversing the response-contingent basis upon which the extinction periods were programmed is made clear in the data from Sessions $136 \cdot 145$. It is apparent that the punishment contingency produced a clear pattern of consistent suppression, replacing the crratic pattern previously exhibited by the noncontingent S1. while the removal of the punishment contin. rency for $S 2$ resulted in a return to the lavel of responding exhibited during the stimulus testing sessions. Table 1, which gives the number and percentage of daily ratios falling above or below .70 . makes char the quantitative similarity in pre. extinction suppression hetwein the effects if noncontingent expoute th extinction and exposure to the stimulus without extinction, while revealing the difference between those two procedures and the exposure to response-contingent extinc. tion.

\section{DISCUSSION}

It is clear that the introduction of extinction conditions in the presence of darkness had an effect on response suppression over and above that of the dark stimulus alone only when the dark-extinction period was made responsecontingent. These results appear to indicate that the preextinction suppression observed in the present study is attributable primarily to passive avoidance of the stimulus associated with nonreinforcement rather than to the typical conditioned emotional response which occurs when response-independent electric shock is made to terminate a previously neutral stimulus (cf. Estes \& Skinner, 1941; Azrin, 1956). The present results with albino rats, along with those of Herrnstein's (1955) low-density reinforcement procedure, support the contention that a period of extinction constitutes an aversive event for a deprived organism, but only if the response decrement can be attributed to the passive avoidance of the presumed aversive stimulus (cf. Dinsmoor, 1954). An alternative explanation of the response decrement observed in both the present study and the Herrnstein study is that the preextinction stimulus actually constituted a discriminative stimulus for extinction, particularly as time elapsed from its onset, since no responses were reinforced in its presence after a specified period of time. This last explanation, however. fails to incorporate the results of Herrnstein's low-density reinforcement procedure in which an increase in responding was observed in the presence of the preextinction warning stimulus.

In considering the results of studies utilizing the CER paradigm witl extinction as the aversive event. the present results support Hermstein's original finding that responding during at preextinction stimulus is determined primarily by the rate of response established on the baseline schedule. Thus. Herrnstein (1955). Ferster (1958). and Leitenberg $(19(3)$, have observed acceleration to a preextinction stimulus when a low-rate producing schedule (VI 2-9 min) was in effect, while Herrnstein (1955) and the present study observed suppression when a high-rate producing schedule (VI .25-.5 min) was in effect. It should be noted, however, that in the cases of Herrnstein and Ferster, the effects observed in the so-called CER paradigm were observed when the extinction period was made contingent upon the first response following a specified duration of warning signal onset. Thus, while Herrnstein argued that CER effects with electric shock were attributable to accidental pairings of responses with shocks, and based his use of the response-contingent extinction procedure in the CER paradigm on that interpretation, the results of the present study suggest that contingency vs noncontingency is an important factor, and that the use of the term "CER paradigm" should be restricted to the noncontingent presentation of an aversive stimulus upon termination of a warning signal. If such a definition is observed strictlv. there have been only three reported CER studies with extinction as the aversive event. The first is the present study, in which no effect of the CER procedure was observed with a high-rate producing schedule. The second is Leitenberg (1966), in which acceleration was observed on a low-rate producing schedule. The third is Leitenberg, Bertsch, \& Coughlin (1968), in which a very unreliable set of results, ranging from minimal suppression to no effect, was observed on a low-rate producing schedule. Thus, while it is clear that responsecontingent extinction can produce either suppression or acceleration during a preextinction warning signal depending on the baseline schedule, further work is necessary to establish the effects of noncontingent extinction in the CER paradigm.

\section{REFIRENCIS}

AZRIN, N. H. Some effects of two intermittent schedules of immediate and nonimmediate punishment. Journal of Psychology, 1956. 42, $3-21$.

DINSMOOR, J, A, Punishment: 1 . The avoidince hypothesis. Psychological Review, 1954, 61. $34-46$.

ISTES, W. K., \& SKINNIR, B. I: Some quantitative properties of anxiety. Joumisl of I.xperimental Psychology. 1941, 29,3901400. I I RSIIR C. B. control of behavior in chimpanzees and pigeons by time-out from positive reinforcement. Psychological Monosaphs: General \& Applied. 1958.72 whole vo. 4611.

11I RRNSIIIN, R, J. Buhavioral conseyuences of the removat of a diseriminative stimulus asiociated with ratiable-interval reinforement. linpublished doctoral dissertation. 1955. Harvard I niversity. 
LEIIENBHRG; H. Conditioned acceleration and conditioned suppression in pigeons. Journal of the Ixperimental Analysis of Bchavior, 1966, $9,205-212$.

LIITENBIRC;, H., BIRTSCH, G. J., \& COUGHLIN, R. C. "Time-out from positive reinforcement" as the UCS in a CER paradigm with rats. Psychonomic Science, 1968, 13, 3-4.
NOTES

1. I his research was supported by NIMH Grant 08430 and by the ('niversity of WisconsinMilwaukee Graduate School.

2. Trenholme, I., Baron, A., \& Kaufman, A. Iffects of signalled time-out from and loss of monetary reinforecment on human operant behavior. Unpublished study.

\section{Schedule-induced nitrogen "drinking" in the rat}

DEREK B. TAYLOR and DA VID LESTER, Center of Alcohol Studies, Rutgers-The State University, New Brunswick, N.J. 08903

Rats working on an FI60 food reinforcement schedule with lick-contingent nitrogen puffs available from a drinking spout exhibited interpellet "drinking" similar to schedule-induced polydipsia. The data were interpreted in terms of a thirst-adventitious reinforcement interaction; the implications for other schedule-induced behaviors were discussed.

Rats bar pressing on an intermittent schedule of food reinforcement drink large quantities of water (schedule-induced polydipsia, SIP) in bursts of drinking after each pellet. Water intake during a 3.17 h session may be three to four times the 24-h normal (Falk, 1961). Another heretof ore unrelated phenomenon is "air drinking" (Hendry \& Rasche, 1961): thirsty rats lick persistently at an air stream or to obtain air puffs contingent upon licking. Our study demonstrates that the conditions producing SIP will also evoke interpellet "air drinking."

\section{METHOD}

The Ss, three experimentally naive, male albino Sprague-Dawley rats, aged 120 to 150 days, weighing 370,400 , and $460 \mathrm{~g}$, were tested in a Grason-Stadler operant chamber enclosed in a sound-attenuated box, containing a lever and a food cup in one wall. A metal drinking spout, wired to record licking, was next to the food cup; its tip, recessed behind a plastic shield, allowed contact only with S's tongue, assuring accurate registration. The spout could deliver nitrogen puffs or water; with nitrogen, contact opened a solenoid valve for $120 \mathrm{msec}$ allowing a flow of $9100 \mathrm{ml} / \mathrm{min}$ of nitrogen, about $18 \mathrm{ml} / \mathrm{puff}$. The operations were programmed with
Digital Equipment Corporation logic. A cumulative recorder provided visual records of licking and reinforcements.

The Ss were given two 20-min sessions in the operant chamber after $23.5 \mathrm{~h}$ of water deprivation, with water available at the spout to learn its location and speed the later development of nitrogen "drinking." Next, the Ss licked for nitrogen during five daily $30-\mathrm{min}$ sessions after $23.5 \mathrm{~h}$ of water deprivation. (Pilot data showed identical performance with nitrogen and air.) During both water and nitrogen training sessions, the bar was inoperable, food being available ad lib in the home cage. Two days of ad lib food and water then followed in the home cage. Body weight was next reduced $20 \%$ by underfeeding and the $S s$ were shaped to bar press for $45-\mathrm{mg}$ food pellets. Nitrogen was available during shaping and subsequent sessions: water, never available in the chamber, was available ad lib in the home cage. After shaping, the Ss were run for one 100-pellet session on CRF, then moved gradually up to Fl60 (sec) during one 120-pellet session. Nine 120-pellet FI60 sessions followed. Home-cage water consumption was measured in the $30-\mathrm{min}$ period preceding and following the FI60 sessions.

\section{RESULTS \\ Rat AD2}

Row 1, Fig. 1, shows a licking record sample from the shaping session. A few long "drinks" are separated by many pellets. As the $S$ moved from CRF to FI60 (Row 2), long "drinks" are more frequently interspersed among many pellets. In the first full session on FI60 (Row 3), interpellet "drinking" suddenly appears, characterized by an alternating sequence of licking and bar pressing, repeated until one of the bar presses yielded a pellet. as if $S$ were pausing during drinking to "test" the bar. Gradually "drinking" bursts lengthened and "bar testing" decreased (Row 4, Session 9 on Fl(60).

\section{Rat AD3}

Rat AD3 did not "drink" during shaping or the CRF sessions. Row 1, Fig. 2, shows the session when $S$ moved from CRF to Fl60. There are occasional "drinks," and a seeming interpellet "drinking" pattern emerges finally. In the first full session on FI60 (Row 2), there is a definite interpellet "drinking" pattern, although with short bursts. "Bar testing" pauses appear, as exhibited by AD2. In the third session (Row 3), "drinking" bursts are longer and "bar testing" diminishes. By the sixth session (Row 4), with the "bar testing" still present, the interpellet "drinking" pattern is fully and permanently established. Rat AD4

Through the first session on FI60, "drinking" developed similarly to that of AD2 with "bar testing" present. However, thereafter "drinking" bursts gradually became short and scattered until "drinking" virtually disappeared.

Water consumption in the home cage during the postsession periods averaged over fourfold that of presession periods. $\mathrm{AD}_{2}$ averaged $1.3 \mathrm{ml}$ during presession periods, $4.3 \mathrm{ml}$ postsession; AD3, 1.4 and $5.3 \mathrm{ml}$; $\mathrm{AD} 4,1.1$ and $6.9 \mathrm{ml}$.

\section{DISCUSSION}

Schedule-induced nitrogen (or air) "drinking" appears almost identical to schedule-induced polydipsia. Falk and others (e.g., Stein, 1964) regard it as a postpellet phenomenon because drinking
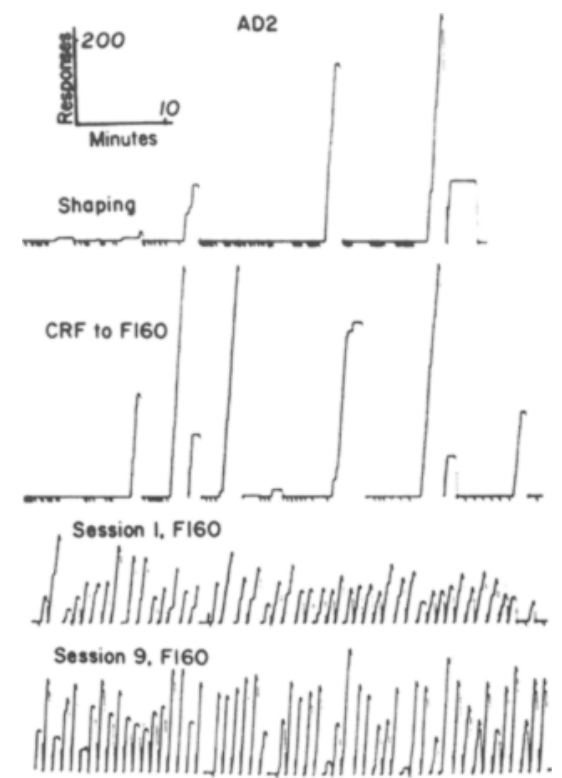

Fig. 1. Lick records for AD2. Each lick steps the recorder cumulatively, resets to baseline by receipt of a pellet. Hatch marks indicate pellets. 